\title{
AVALIAÇÃO DE DEFENSIVOS NATURAIS PARA O CONTROLE DE Tetranychus abacae BAKER \& PRITCHARD (ACARI: TETRANYCHIDAE) EM FLORES TROPICAIS
}

\author{
Renata Maria Vital Santos ${ }^{1}$, Aloyséia Cristina da Silva Noronha ${ }^{2}$, Fábio Mathias Corrêa ${ }^{3}$, \\ Maria Aparecida Leão Bittencourt ${ }^{4}$ \\ ${ }^{1}$ Programa de Pós-graduação em Produção Vegetal, PPGPV/UESC, Rod. Ilhéus - Itabuna, km 16, 45662-900, Ilhéus, \\ Bahia, Brasil, renatavitalagro@gmail.com \\ ${ }^{2}$ Embrapa Amazônia Oriental, TV. Enéas Pinheiro, s/n, Pav. Pesquisa, 66.095-100, Belém, Pará, Brasil, \\ aloyseia@cpatu.embrapa.br \\ ${ }^{3}$ Departamento de Ciências Exatas - DCET/UESC, Rod. Ilhéus - Itabuna, km 16, 45662-900, Ilhéus, Bahia, Brasil, \\ fmcron@gmail.com \\ ${ }^{4}$ Departamento de Ciências Agrárias e Ambientais - DCAA/UESC, Rod. Ilhéus - Itabuna, km 16, 45662-900, \\ Ilhéus, Bahia, Brasil, malbitte@gmail.com
}

\section{RESUMO}

Defensivos alternativos, como caldas e extratos vegetais, são promissores no controle de ácaros fitófagos, pois visam contribuir para a diminuição no uso intenso de agrotóxicos. $\mathrm{O}$ ácarovermelho, Tetranychus abacae Baker \& Pritchard, é relatado nos seguintes hospedeiros Heliconia bihai e H. psittacorum (Heliconiaceae), Etlingera elatior (Zingiberaceae) e Musa acuminata (Musaceae). Em razão de não existir registro de produtos químicos para o controle de pragas em flores tropicais, o objetivo do presente trabalho foi avaliar a ação acaricida da 'calda-de-fumo' (Nicotiana tabacum L.), da calda de 'pimenta-malagueta' (Capsicum frutescens L.) e da 'manipueira' (Manihot esculenta Crantz) sobre T. abacae nas concentrações de 25\%, 50\% e 75\%. Cada unidade experimental foi constituída de uma placa de Petri contendo um disco de folha de helicônia, com 20 fêmeas do ácaro na sua face ventral. Foi avaliada a ação de contato (imersão dos discos de folhas nos tratamentos por 10 segundos) e a ação de pulverização (borrifador manual); as avaliações para mortalidade foram realizadas até cinco horas após a aplicação dos tratamentos, com observações a cada 60 minutos. Foi observada interação entre o modo de ação do produto, tempo de exposição e a concentração utilizada. Houve efeito significativo do aumento da concentração e percentual de ácaros mortos. A 'calda-de-pimenta' e a 'manipueira', causaram mortalidade de $100 \%$ dos ácaros, por ação de contato, após a primeira hora, sendo que para os demais tratamentos e forma de aplicação, a mortalidade de aproximadamente $100 \%$ dos ácaros ocorreu somente após cinco horas da aplicação. O efeito acaricida, por ação de contato, dos tratamentos foi observado com $75 \%$ de concentração e maior tempo de exposição. Os resultados sugerem que os produtos naturais podem ser uma alternativa no controle de T. abacae, principalmente em plantios de helicônias.

Palavras-chave: Ácaro-vermelho, bioinseticidas, floricultura 


\title{
EVALUATION OF NATURAL PRODUCTS FOR THE CONTROL OF Tetranychus abacae BAKER \& PRITCHARD (ACARI: TETRANYCHIDAE) ON TROPICAL FLOWERS.
}

\begin{abstract}
Defense alternatives, like syrups and vegetable extracts, are promising to control phytophagous mites, contributing to the reduction of intense pesticide use. The red mite, Tetranychus abacae Baker \& Pritchard, is related to the following hosts: Heliconia bihai and $H$. psittacorum (Heliconiaceae), Etlingera elatior (Zingiberaceae) and Musa acuminata (Musaceae). Because there are no chemical products registered control for tropical flowers, this study evaluated the effect of 'calda-de-fumo' (Nicotiana tabacum L.), “'pickle-pepper" syrup (Capsicum frutescens L.) and 'manipueira' (Manihot esculenta Crantz) on red mites at concentrations of $25 \%, 50 \%$ and $75 \%$. Each experimental unit was made up of a Petri dish containing a heliconia leaf disc, with 20 mite females on its ventral side. The leaves were inserted in the treatment for 10 seconds, observing the contact action and pulverized liquids (Vortex mixer). The mortality evaluations were observed for five hours after the treatment application. Mortality was counted every 60 minutes. Interaction was observed between the mode of action of the product, exposure time and the concentration used. There was significant effect of increase of concentration and percentage of mites killed. The "pickle-pepper" and "manipueira", caused mortality of $100 \%$ of mites, by contact action, after the first hour, and for other treatments and form of implementation, the mortality rate of approximately $100 \%$ of mites occurred only after five hours of application. The effect of mitecide, by contact action, of treatments was observed with $75 \%$ concentration and exposure time. The results suggest that natural products can be an alternative in the control of T. abacae, mainly in heliconia plantations.
\end{abstract}

Keywords: Red mites, bioinsecticides, floriculture

\section{INTRODUÇÃO}

O ácaro-vermelho Tetranychus
abacae Baker \& Pritchard (Acari: Tetranychidae) possui alguns hospedeiros conhecidos, pertencentes às famílias Musaceae e Heliconiaceae. No estado de Santa Catarina a espécie está associada à Musa acuminata; em São Paulo à Heliconia bihai e M. acuminata; e em Pernambuco, foi associada à Musa sp., $H$. bihai e $H$. psittacorum (Flechtmann 1996; Assis et al., 2002; Vasconcelos et al., 2004). Há relato desta espécie em bastão-do-imperador (Etlingera elatior - Zingiberaceae), em alpíneas (Alpinia purpurata - Zingiberaceae) e em $H$. bihai, principalmente em períodos de temperatura elevada (Warumby et al., 2004).
Os ácaros-vermelhos provocam manchas nas folhas de espécies de flores tropicais, as quais perdem o brilho característico, tornando-se bronzeadas e recobertas por teia, que pode ser vista facilmente na face inferior do limbo foliar. Em população elevada, as teias podem ligar folhas diferentes da mesma planta, na região apical; inicialmente ocorre uma clorose, progredindo para secamento e queda prematura das folhas (Flechtmann, 1985; Assis et al., 2002; Warumby et al., 2004; Moraes \& Flechtmann 2008).

Atualmente, não existem produtos químicos registrados pelo Ministério da Agricultura, Pecuária e Abastecimento (MAPA) para o controle de pragas em helicônias. Isso tem feito com que os produtores utilizem indiscriminadamente 
agrotóxicos registrados para outras culturas, propiciando riscos à saúde humana e ao meio ambiente. Esses problemas podem ser miminizados com o emprego de métodos alternativos de controle, os quais têm surgido como uma ferramenta importante no manejo de pragas. São poucos os estudos sobre o efeito de extratos botânicos sobre ácaros de importância econômica (Gonçalves et al., 2001a; Gonçalves et al., 2001b; Sacramento et al., 2006; Antonious et al., 2006; Brito et al., 2006; Pontes et al., 2007).

Com o objetivo de fornecer informações a serem utilizadas para o desenvolvimento de métodos alternativos no controle de pragas, em cultivos de flores tropicais, neste trabalho foi avaliada a ação acaricida da 'calda-de-fumo' (Nicotiana tabacum L.), da calda de 'pimentamalagueta' (Capsicum frutescens L.) e da 'manipueira' subproduto da produção de farinha de mandioca (Manihot esculenta Crantz), sobre T. abacae.

\section{MATERIAL E MÉTODOS}

O trabalho foi realizado do Laboratório de Entomologia da Universidade Estadual de Santa Cruz (UESC) em Ilhéus, Bahia. Foram realizados bioensaios de concentração-mortalidade com fêmeas adultas de $T$. abacae coletadas em mudas de $H$. bihai, sem pulverização com produto químico, em casa-de-vegetação da UESC. O potencial acaricida da 'calda-de-fumo', da calda de 'pimenta-malagueta' e da 'manipueira', foi avaliado pela ação de contato e ação de pulverização. A 'calda-defumo' foi preparada utilizando-se $20 \mathrm{~cm}$ de fumo-de-rolo, o qual foi repicado e colocado em solução de álcool etílico a 50\% (500 mL de água $+500 \mathrm{~mL}$ de álcool), permanecendo em repouso por 48 horas (Azevedo \& Gurgel, 2006). Para o preparo da calda de 'pimenta-malagueta', $300 \mathrm{~g}$ de frutos de pimenta-malagueta (sem o talo) foram triturados em liquidificador, misturados a dois litros de água e $15 \mathrm{~g}$ de sabão em pó, e depois coados em tecido. A 'manipueira' foi obtida em uma propriedade rural de Ilhéus, Bahia, extraída da variedade de mandioca conhecida como 'manteiga', esta foi coada e diluída em água conforme cada concentração, sendo utilizada no mesmo dia em que foi extraída.

Os tratamentos utilizados nos bioensaios constaram das 'caldas' do fumo e da pimenta, e da 'manipueira', diluídas em água destilada nas concentrações $25 \%, 50 \%$ e $75 \%$, e na testemunha foi aplicado água destilada.

Para avaliar a ação de contato, discos de folhas de $H$. bihai foram imersos nos tratamentos por 10 segundos, agitados levemente e colocados para secar em condições ambiente durante 30 minutos, antes da transferência das fêmeas. Os tratamentos foram pulverizados, por meio de borrifador manual (Giolo et al., 2005), para cobertura dos discos de folhas, com aproximadamente $9 \mathrm{~mL}$ do líquido em cada disco de folha.

A parcela experimental foi constituída de uma placa de Petri $(9 \mathrm{~cm}$ de diâmetro), contendo duas camadas de papel filtro umedecido com água destilada, sobre o qual foi colocado um disco de folha de helicônia com três centímetros de diâmetro, circundado com algodão hidrófilo para evitar a fuga dos ácaros. Cada disco de folha recebeu 20 fêmeas adultas de T. abacae e as placas foram vedadas com filme plástico de PVC, nos quais foram feitos pequenos furos para aeração; as placas foram mantidas em câmara climática do tipo BOD, a $25 \pm 1^{\circ} \mathrm{C}$ e fotofase de 12 horas.

A mortalidade das fêmeas de $T$. abacae foi avaliada após 1, 2, 3, 4 e 5 horas após a aplicação dos tratamentos, sendo considerados mortos os ácaros que foram tocados levemente, com pincel de cerdas bem finas, e não se movimentavam até a distância equivalente ao comprimento do seu 
corpo (Mourão et al., 2004). O delineamento experimental utilizado foi inteiramente casualizado, em cinco repetições. Os dados foram submetidos à análise de regressão linear múltipla, sendo utilizado o programa estatístico R 2.12.2 (R Development Core Team 2011).

\section{RESULTADOS E DISCUSSÃO}

Os resultados da regressão linear múltipla entre os tratamentos, as concentrações e modo de ação estão apresentados na Tabela 1. A mortalidade (\%) das fêmeas de $T$. abacae observada em relação à concentração (\%) dos diferentes tratamentos e horas após a aplicação obtidas através das equações de regressão estão apresentadas na Figura 1.

Foi observada interação entre o modo de ação do produto, tempo de exposição e a concentração utilizada. Houve efeito significativo do aumento da concentração no percentual de ácaros mortos. Com a 'caldade-pimenta' e a 'manipueira', a mortalidade de $100 \%$ dos ácaros ocorreu na primeira hora após aplicação dos tratamentos por ação de contato, sendo que para os demais tratamentos e forma de aplicação, a mortalidade de aproximadamente $100 \%$ dos ácaros ocorreu somente após 5 horas de aplicação (Figura 1).

A 'calda-de-fumo' provocou maior mortalidade de fêmeas na primeira hora, por ação de contato e de pulverização (Figura 1A), provavelmente em função da ação da nicotina, que apresenta ação de choque (contato e ingestão), e é totalmente degradada em 24 horas, não deixando qualquer resíduo tóxico (Moreira et al., 2006; Dequech et al., 2008).

A ação da 'calda-de-pimenta' (Figura 1B) sobre os ácaros pode ser resultante da presença do alcalóide capsaicina, responsável pela pungência, que também pode provocar o efeito repelência (Procópio et al., 2003a, 2003b; Antonious et al., 2006).

A 'manipueira' aplicada em pulverização, nas concentrações testadas causou $100 \%$ de mortalidade, após uma hora da aplicação (Figura 1C), provavelmente devido ao principio ativo o ácido cianídrico, demonstrando seu potencial no controle de pragas conforme relatado em outros estudos (Ponte et al., 1988; Ponte et al., 1996; Ponte 2000; Gonzaga et al., 2007; Mendes et al., 2007; Gonzaga et al., 2008).

Tabela 1. Modelos de regressão linear ajustado para os fatores significativos a $5 \%$ de probabilidade, do efeito de tratamentos e modo de aplicação 'in vitro' sobre fêmeas de Tetranychus abacae.

\begin{tabular}{lcc}
\hline \multicolumn{1}{c}{ Tratamento } & Ação & Equação \\
\hline 'Calda-de-fumo' & Contato & $\mathrm{Y}=4.66+20.24 *$ tempo $-2.28 *$ tempo^ $2+0.84 *$ conc. \\
'Calda-de-fumo' & Pulverização & $\mathrm{Y}=1.13+17.76 *$ tempo $-2.16 *$ tempo^2+0.98*conc. \\
'Calda-de-pimenta' & Contato & $\mathrm{Y}=24.76+4.23 *$ tempo $+1.18 *$ conc. \\
'Calda-de-pimenta' & Pulverização & $\mathrm{Y}=88.33+1.6 *$ tempo $+0.072 *$ conc. \\
Manipueira & Contato & $\mathrm{Y}=41.73+33.02 *$ tempo $-3.71 *$ tempo $2+0.94 *$ conc. \\
Manipueira & Pulverização & $\mathrm{Y}=100-1.9 \mathrm{E} 14 *$ tempo $-1.1 \mathrm{E} 15 *$ conc. \\
\hline
\end{tabular}



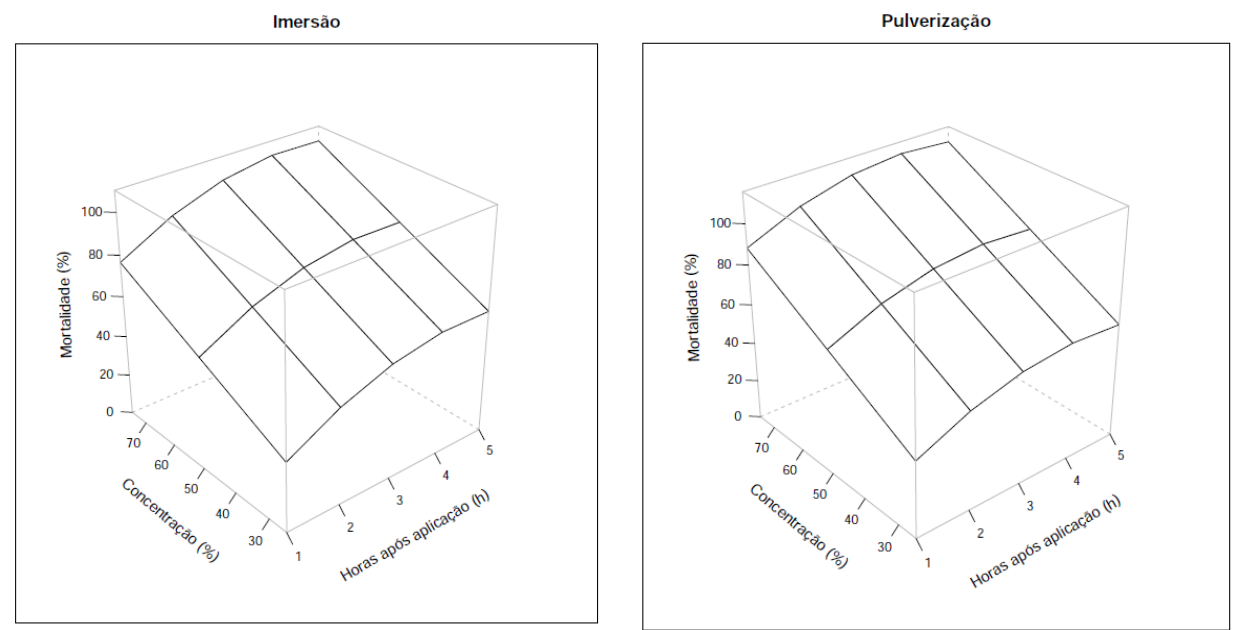

A: Tratamento com 'calda-de-fumo'
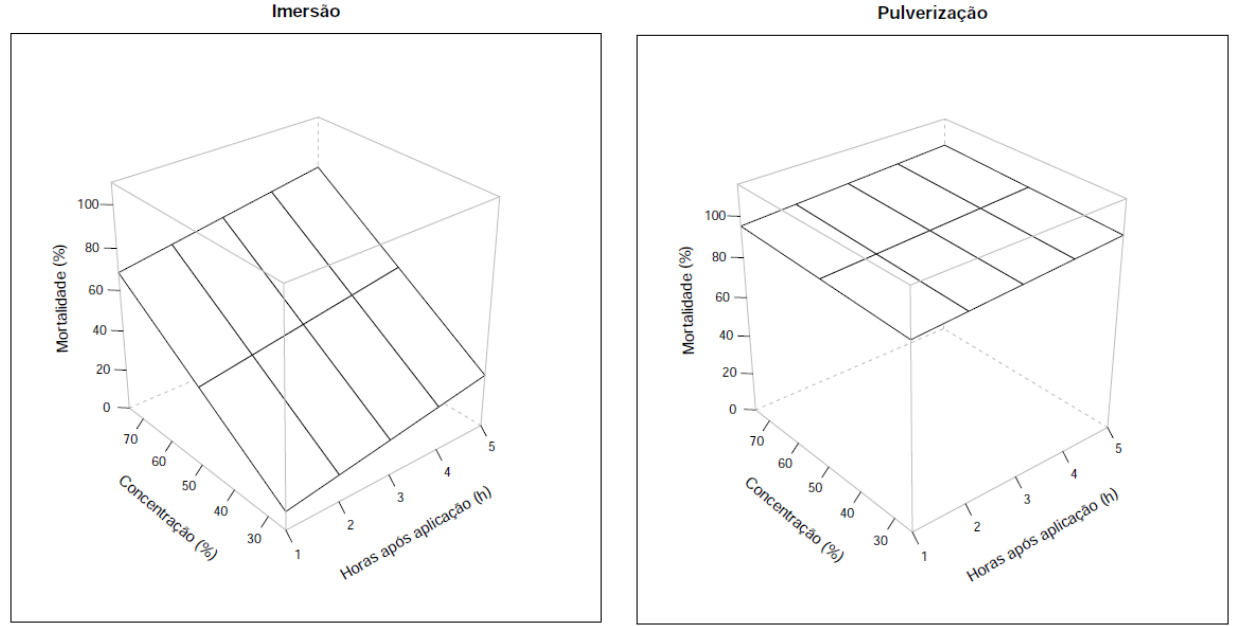

B: Tratamento com 'calda-de-pimenta' Imersão
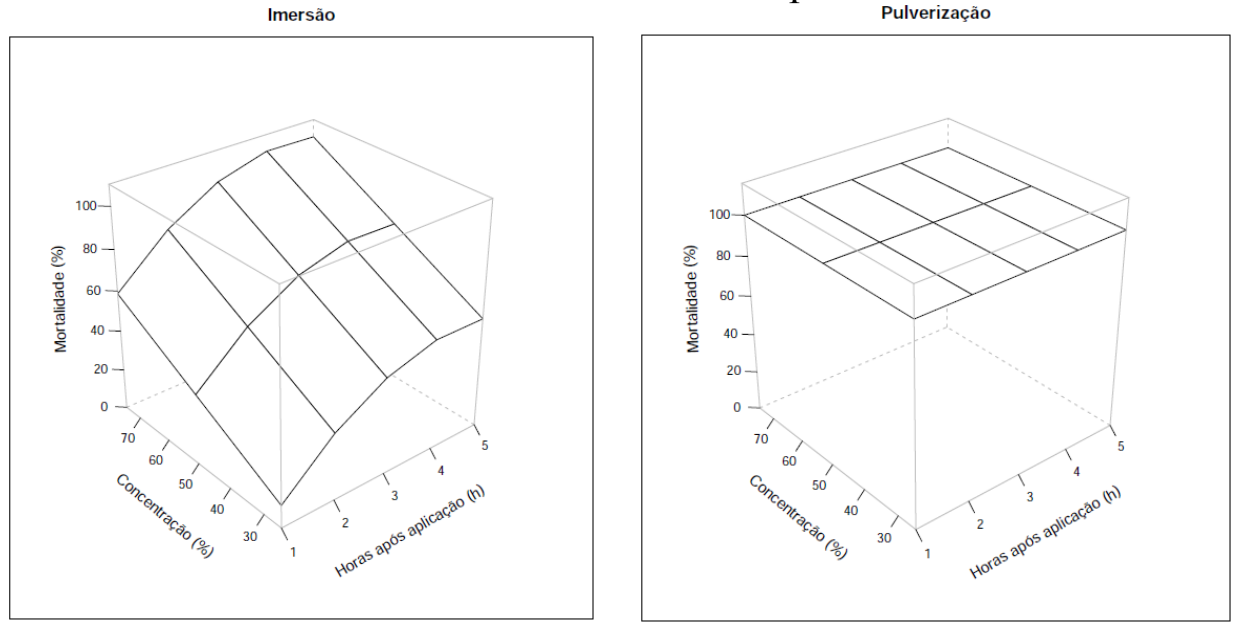

C: Tratamento com 'manipueira'

Figura 1. Mortalidade (\%), 'in vitro', de fêmeas de Tetranychus abacae, sob ação de diferentes tratamentos, a $25 \%, 50 \%$ e $75 \%$ de concentração. 


\section{CONCLUSÃO}

A 'calda-de-pimenta' e a 'manipueira' a 25\% aplicadas sob pulverização apresentam efeito acaricida sobre fêmeas de Tetranychus abacae, por ação de pulverização.

A aplicação dos produtos acaricidas testados, sob a forma de contato requer uma maior concentração $(75 \%)$ e maior tempo de exposição para apresentar efeito acaricida.

Produtos naturais podem ser uma alternativa no controle de ácaros fitófagos.

\section{AGRADECIMENTOS}

À Fundação de Amparo à Pesquisa do Estado da Bahia - FAPESB, pela concessão de bolsa ao primeiro autor e suporte financeiro, à Universidade Estadual de Santa Cruz - UESC onde foi realizado o trabalho, e aos produtores de flores tropicais, que gentilmente cederam seus cultivos para realização das coletas.

\section{REFERÊNCIAS BIBLIOGRÁFICAS}

ANTONIOUS, G.F.; MEYER, J.E.; SNYDER, J.C. 2006. Toxicity and repellency of hot pepper extracts to spider mite, Tetranychus urticae Koch. Journal of Environmental Science and Health B, 41:1383-1391.

ASSIS, S.M.P.; MARIANO, R.L.R.; GONDIM JUNIOR, M.G.C.; MENEZES, M.; ROSA, R.C.T. 2002. Doenças e pragas de helicônias. Diseases and pests of heliconias. Recife: UFRPE, $102 \mathrm{p}$.

AZEVEDO, F. R.; GURGEL, L. S. 2006. Ação da nicotina para o controle da mosca-branca Bemisia tabaci biótipo B em meloeiro. In: Anais do III Congresso Brasileiro de Defensivos Agrícolas Naturais - III COBRADAN - Belém - PA, p.158, Embrapa/Sebrae.
DEQUECH, S.T.B.; SAUSEN, C. D., LIMA, C. G.; EGEWARTH, R. 2008. Efeito de extratos de plantas com atividade inseticida no controle de Microtheca ochroloma Stal (Col.: Chrysomelidae), em laboratório. Biotema, 21: 41-46.

FLECHTMANN, C. H. W. 1985. Ácaros de importância agrícola. São Paulo: Nobel, 189p.

FLECHTMANN, C. H. W. 1996. Rediscovery of Tetranychus abacae Baker \& Pritchard, additional description and notes on South American spider mites (Acari, Prostigmata, Tetranychidae). Revista Brasileira de Zoologia, 13: 569-578.

GIOLO, F. P.; GRÜTZMACHER, A. D.; MANZONI, C. G.; FACHINELLO, J. C.; NÖRNBERG, S. D.; STEFANELLO JÚNIOR, G. J. 2005. Seletividade de agrotóxicos indicados na produção integrada de pêssego a Trichogramma pretiosum Riley, 1879 (Hymenoptera: Trichogrammatidae).

Revista

Brasileira de Fruticultura, 27: 222225.

GONZAGA, A. D.; SOUZA, S.G.A.; PYDANIEL, V.; RIBEIRO, J.D. 2007. Potencial de manipueira de mandioca (Manihot esculenta Crantz) no controle do pulgão-preto de citros (Toxoptera citricida Kirkaldy, 1907). Revista Brasileira de Agroecologia, 2: 646650.

GONZAGA, A. D.; GARCIA, M.V.B.; SOUSA, S.G.A.; PY-DANIEL, V.; CORREA, R.S.; RIBEIRO, J.D. 2008. Toxicity of cassava manipueira (Manihot esculenta Crantz) and erva-derato (Palicourea marcgravii St. Hill) to adults of Toxoptera citricida Kirkaldy (Homoptera: Aphididae). Acta Amazonica, 38: 101-106.

MENDES, P.C.D.; AMBROSANO, E.J.; GUIRADO, N.; ROSSI, F.; ARÉVALO, R.A. 2007. Utilização de defensivos 
alternativos no controle de Enneothrips flavens (Thysanoptera: Thripidae) na cultura do amendoim. Revista Brasileira de Agroecologia, 2: 584586.

MORAES, G.J.; FLECHTMANN, C.H.W. 2008. Manual de acarologia: acarologia básica e ácaros de plantas cultivadas no Brasil. Ribeirão Preto: Holos, 308p.

MOREIRA, M. D.; PICANÇO, M. C.; SILVA, E. M.; MORENO, S. C.; MARTINS, J. C. 2006. Uso de inseticidas botânicos no controle de pragas. In: Venzon, M., Paula Junior, T.J., Pallini, A. Controle alternativo de pragas e doenças. Viçosa: EPAMIG/ CTZM: UFV, cap. 5, p. 89-120.

MOURÃO, S. A.; SILVA, J. C. T.; GUEDES, R. N. C.; VENZON, M.; JHAM, G. N.; OLIVEIRA, C. L.; ZANUNCIO, J. C. 2004. Seletividade de extratos de nim (Azadirachta indica A. Juss.) ao ácaro predador Iphiseiodes zuluagai (Denmark \& Muma) (Acari: Phytoseiidae)Neotropical Entomology, 33: 613-617.

PONTE, J. J. 1996. Eficiência da manipueira no controle de ácaro-branco do mamoeiro. Revista Agricultura, 71: 259-261.

PONTE, J. J. 2000. Uso da Manipueira como insumo agrícola: defensivo e fertilizante. In: CEREDA, M. P. (Coord.). Culturas de tuberosas amiláceas latino americanas: Uso, manejo e tratamento de subprodutos da industrialização da mandioca. São Paulo: Fundação Cargill. p. 80-95.

PONTE, J. J.; FRANCO, A.; SANTOS, J. H. R. 1988. Teste preliminar sobre a utilização da manipuiera como inseticida. Revista Brasileira de Mandioca, 7: 89-90.

PROCÓPIO, S. O; VENDRAMIM, J.D.; RIBEIRO JÚNIOR, J.I.; SANTOS, J.B. 2003a. Bioatividade de diversos pós de origem vegetal em relação à Sitophilus zeamais Mots (Coleoptera: Curculionidae). Ciência Agrotecnologia, 27: 1231-1236.

PROCÓPIO, S. O.; VENDRAMIM, J.D.; RIBEIRO JÚNIOR, J.I.; SANTOS, J.B. 2003b. Efeito de pós vegetais sobre Acanthoscelides obtectus (Say) e Zabrotes subfasciatus (Boh.) (Coleoptera: Bruchidae). Revista Ceres, 50: 395-405.

R 2.12.2 Development Core Team 2011. R: A language and environment for statistical computing. $R$ Foundation for Statistical Computing, Vienna, Austria. URL. Disponível em: <http://www.r-project.org>. Acesso em: 11 abr. 2011.

VASCONCELOS, G. J. N.; SILVA, F. R.; GONDIM JR, M.G.C.; BARROS, R.; OLIVEIRA, J.V. 2004. Efeito de diferentes temperaturas no desenvolvimento e reprodução de Tetranychus abacae Baker \& Printchard (Acari: Tetranychidae) em bananeira Musa sp. cv. Prata. Neotropical Entomology, 33: 149-154.

WARUMBY, J.F.; COELHO, R. S. B.; LINS, S. R. O. 2004. Principais doenças e pragas em flores tropicais no Estado de Pernambuco. Recife: SEBRAE, 98p. 